\title{
Testing Bell Inequalities at the LHC with Top-Quark Pairs
}

\author{
M. Fabbrichesi, ${ }^{1}$ R. Floreanini, ${ }^{1}$ and G. Panizzo $\circledast^{2}$ \\ ${ }^{1}$ INFN, Sezione di Trieste, Via Valerio 2, 34127 Trieste, Italy \\ ${ }^{2}$ Dipartimento Politecnico di Ingegneria ed Architettura, Università degli Studi di Udine, Via della Scienze 206, 33100 Udine, Italy \\ and INFN, Sezione di Trieste (Gruppo Collegato di Udine), via delle Scienze, 208, 33100 Udine, Italy
}

(Received 17 February 2021; accepted 21 September 2021; published 15 October 2021)

\begin{abstract}
Correlations between the spins of top-quark pairs produced at a collider can be used to probe quantum entanglement at energies never explored so far. We show how the measurement of a single observable can provide a test of the violation of a Bell inequality at the 98\% C.L. with the statistical uncertainty of the data already collected at the Large Hadron Collider, and at the $99.99 \%$ C.L. with the higher luminosity of the next run. Detector acceptance, efficiency, and migration effects are taken into account. The test relies on the spin correlations alone and does not require the determination of probabilities - in contrast to all other tests of Bell inequalities.
\end{abstract}

DOI: 10.1103/PhysRevLett.127.161801

Introduction.-A characteristic property of a quantum system is the presence of quantum correlations (entanglement) among its constituents not accounted for by classical physics (for a review, see [1]), leading to the violation of specific constraints, the so-called Bell inequalities [2,3]. The violation of Bell inequalities requires the presence of the strongest version of quantum nonlocality; although weaker forms of nonclassical correlations have been identified, they play no role in our considerations.

Quantum correlations can readily be studied in a bipartite system made of two spin-1/2 particles [4]. This physical system is routinely produced at colliders and the spin correlations among quark pairs have been shown $[5,6]$ to be a powerful tool in the physical analysis - limited aspects of which have been already studied by the experimental collaborations at the LHC on data at 7 [7], 8 [8], and $13 \mathrm{TeV}$ [9] of center-of-mass (c.m.) energy.

In this Letter, we focus on top-antitop pairs produced at the Large Hadron Collider (LHC) and identify a single observable probing the presence of quantum correlations among their spins. The measurement of such an observable provides a test of a (generalized) Bell inequality.

Many experiments have been performed to test analogous inequalities in various quantum systems involving photons and atoms $[4,10-13]$. Similar tests in the highenergy regime of particle physics have been suggested by means of $e^{+} e^{-}$collisions [14], neutral meson physics $[15,16]$, positronium [17], charmonium decays [18], and

Published by the American Physical Society under the terms of the Creative Commons Attribution 4.0 International license. Further distribution of this work must maintain attribution to the author(s) and the published article's title, journal citation, and DOI. Funded by SCOAP ${ }^{3}$. neutrino oscillations [19]. No test has so far been performed at the high energies made available by the LHC - even though some preliminary work has been done in $[5,6]$ and more recently in [20]. In particular, we build on the results of [20] in which the entanglement of the top-quark pairs and the kinematical regions where it could be maximal were identified and explicitly discussed.

Let us stress that all these tests involve the direct measurement of the joint probabilities entering the various inequalities and therefore might be affected by the so-called loopholes, depending on the specific characteristics of the used setups. Our approach is quite different and unexplored: the focus is not on the probabilities of joint events, specifically top-quark pair spin projection measurements, but rather on their mutual spin correlations. Such a measurement of correlations will provide evidence against a whole class of local completions of quantum mechanics by explicitly exposing their internal inconsistency. In order to be validated, these classical theories will need to reproduce both the probabilities entering the Bell inequality and the averages of the spin correlation matrix through the presence of auxiliary stochastic variables and do that both at atomic energies and in the extreme relativistic setting of proton collisions at the LHC.

Reformulating the actual determination of the selected spin observable into a statistical test, we show how the value of this observable can be extracted from the events and the violation quantified at the confidence level (C.L.) of $98 \%$ with the data already collected by the experimental collaborations at the LHC and $99.99 \%$ C.L. (4 $\sigma$ significance) with the higher luminosity of the next run. Detector acceptance, efficiency, and migration effects have been taken into account.

Methods.-The quantum state of a two spin-1/2 pair, as the one formed by a top-quark pair system, can be expressed by the density matrix 


$$
\begin{aligned}
\rho= & \frac{1}{4}\left[\mathbb{1} \otimes \mathbb{1}+\sum_{i} A_{i}\left(\sigma_{i} \otimes \mathbb{1}\right)+\sum_{j} B_{j}\left(\mathbb{1} \otimes \sigma_{j}\right)\right. \\
& \left.+\sum_{i j} C_{i j}\left(\sigma_{i} \otimes \sigma_{j}\right)\right]
\end{aligned}
$$

where $\sigma_{i}$ are Pauli matrices, $\mathbb{1}$ is the unit $2 \times 2$ matrix, while the sums of the indices $i, j$ run over the labels representing any orthonormal reference frame in three dimensions. The real coefficients $A_{i}=\operatorname{Tr}\left[\rho\left(\sigma_{i} \otimes \mathbf{1}\right)\right]$ and $B_{j}=\operatorname{Tr}[\rho(\mathbf{1} \otimes$ $\left.\left.\sigma_{j}\right)\right]$ represent the polarization of the two spins, while the real matrix $C_{i j}=\operatorname{Tr}\left[\rho\left(\sigma_{i} \otimes \sigma_{j}\right)\right]$ gives their correlations. In the case of the top-quark pair system, $A_{i}, B_{j}$, and $C_{i j}$ are functions of the parameters describing the kinematics of the quark pair production.

In the c.m. reference frame of the top-quark pair system as produced at a $p p$ collider, the two spin- $1 / 2$ quarks fly apart in opposite directions. One can then extract the probability $\mathcal{P}\left(\uparrow_{\hat{n}} ;-\right)$ of finding the spin of one quark in the state $\uparrow_{\hat{n}}$, with the projection of the spin along the axis determined by the unit vector $\hat{n}$ pointing in the up direction. Similarly, one can consider double probabilities, like $\mathcal{P}\left(\uparrow_{\hat{n}} ; \downarrow_{\hat{m}}\right)$, of finding the projection of the spin of the quark along the unit vector $\hat{n}$ pointing in the up state, while the companion antiquark has the projection of its spin along the direction of a different unit vector $\hat{m}$ pointing in the down state.

In classical physics, these probabilities involve averages over suitable distributions of variables and obey the following (generalized) Bell inequality [11]:

$$
\begin{aligned}
& \mathcal{P}\left(\uparrow_{\hat{n}_{1}} ; \uparrow_{\hat{n}_{2}}\right)-\mathcal{P}\left(\uparrow_{\hat{n}_{1}} ; \uparrow_{\hat{n}_{4}}\right)+\mathcal{P}\left(\uparrow_{\hat{n}_{3}} ; \uparrow_{\hat{n}_{2}}\right)+\mathcal{P}\left(\uparrow_{\hat{n}_{3}} ; \uparrow_{\hat{n}_{4}}\right) \\
& \quad \leq \mathcal{P}\left(\uparrow_{\hat{n}_{3}} ;-\right)+\mathcal{P}\left(-; \uparrow_{\hat{n}_{2}}\right),
\end{aligned}
$$

where $\hat{n}_{1}, \hat{n}_{2}, \hat{n}_{3}$, and $\hat{n}_{4}$ are four different three-dimensional unit vectors determining four spatial directions along which the spins of the quark and antiquark can be measured. In quantum mechanics the same probabilities are computed as expectation of suitable spin-observable operators in the state (1), so that the previous inequality reduces to the following constraint:

$$
\left|\hat{n}_{1} \cdot C \cdot\left(\hat{n}_{2}-\hat{n}_{4}\right)+\hat{n}_{3} \cdot C \cdot\left(\hat{n}_{2}+\hat{n}_{4}\right)\right| \leq 2,
$$

involving only the spin correlation matrix $C_{i j}$ and not the polarization coefficients $A_{i}$ and $B_{j}$.

In order to test the Bell inequality in Eq. (3), one needs to experimentally determine the matrix $C$ and then suitably choose four spatial directions $\hat{n}_{1}, \hat{n}_{2}, \hat{n}_{3}$, and $\hat{n}_{4}$ that maximize the left-hand side of (3). In practice, there is no need to optimize the choice of $\hat{n}_{i}$ : this maximization process has already been performed in full generality in Ref. [12], for a generic spin correlation matrix. Indeed, consider the matrix $C$ and its transpose $C^{T}$ and form the symmetric, positive, $3 \times 3$ matrix $M=C^{T} C$ whose three eigenvalues $m_{1}, m_{2}, m_{3}$ can be ordered by decreasing magnitude: $m_{1} \geq m_{2} \geq m_{3}$. The two-spin state density matrix $\rho$ in (1) violates the inequality (3), or equivalently (2), if and only if the sum of the two greatest eigenvalues of $M$ is strictly larger than 1 , that is

$$
m_{1}+m_{2}>1 \text {. }
$$

In other words, given a spin correlation matrix $C$ of the state $\rho$ that satisfies (4), there are for sure choices for the vectors $\hat{n}_{1}, \hat{n}_{2}, \hat{n}_{3}, \hat{n}_{4}$ for which the left-hand side of (3) is larger than 2 .

It should be stressed that the above formulation, based on the relation (2), departs from the more standard approaches adopted in testing Bell inequalities, in particular in quantum optics. While in the standard, direct tests one needs to experimentally determine the expectation values of spin observables entering the Bell inequalities, in the above, indirect approach the actual measure of probabilities is avoided, in favor of the determination of the spin correlation matrix $C$ - the entries of which can be measured by studying the kinematics of the quark-antiquark decay products [6].

In the recent analysis [9], the spin correlations of the topquark pairs produced at the LHC are analyzed but only after being averaged over a large portion of phase space; the values obtained for the entries of $C$ are in agreement with the inequality (3), for any choice of the four vectors $\hat{n}_{i}$. This agreement is the consequence of the averaging procedure (mixing) that unavoidably reduces the entanglement content of the density matrix $\rho$.

On the other hand, the study in [20] suggests that by focusing on specific, small regions of the phase space, the entanglement of the top-quark pair state could be close to maximal (see also [21]) and the Bell inequality in Eq. (2) could be violated at the maximal level.

Results.-The sum of the eigenvalues $m_{1}+m_{2}$ provides an observable whose value, as extracted from the data, tests whether the Bell inequality in Eq. (3) is violated or not. To compute this observable we collect all the entries of the correlation matrix $C$ as given in the process

$$
p p \rightarrow t+\bar{t} \rightarrow \ell^{ \pm} \ell^{\mp}+\text { jets }+E_{T}^{\text {miss }},
$$

where $\ell=e, \mu$ are taken only in different-flavor combinations, in order to better connect with experimental measurements in this channel. $E_{T}^{\text {miss }}$ stands for the transverse missing energy.

We simulate full matrix elements for the top quark production and decays through the decay chain formalism built into MADGRAPH5 [22], which embeds full spin correlations and Breit-Wigner effects, thus excluding only nonresonant diagrams. Within the standard model we consider gluon $(g g)$ and quark $(q \bar{q})$ initiated top-quark pair production at leading order in the strong and electroweak couplings, using the NNPDF23 [23] leading order parton 


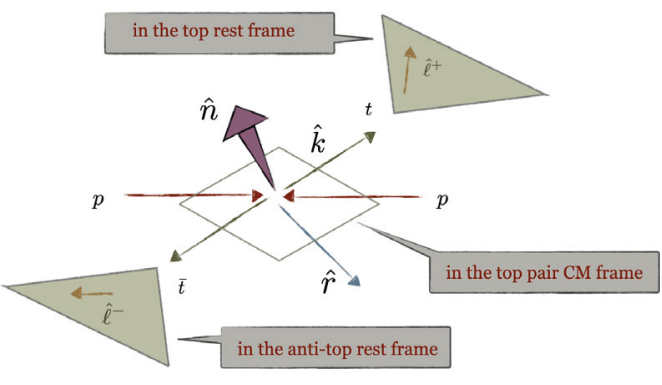

FIG. 1. Kinematics and coordinate systems used in the analysis. The $t$ and $\bar{t}$ rest frames are reached from the $t \bar{t}$ center of mass frame by rotation-free boosts.

distributions set and within the four flavor number scheme, thus fully taking into account for bottom quark mass effects. Next-to-leading order corrections in the strong coupling are known to be small on the largest entries of $C$ : at the LHC energies their impact is less than $2 \%$ [5], and will be neglected in the following. We assume a c.m. energy of $13 \mathrm{TeV}$, setting both the renormalization and factorization scales to the sum of the transverse energies of the finalstate particles.

We follow [6] for the choice of orthonormal basis for the matrix $C$ of Eq. (1). The unit vectors $\hat{r}$ and $\hat{n}$ are built starting from the direction of flight $\hat{k}$ of the top quark in the top pair c.m. frame with respect to one of the proton beams directions in the laboratory frame $\hat{p}$ :

$$
\hat{p}=(0,0,1), \quad \hat{r}=\frac{1}{r}(\hat{p}-y \hat{k}), \quad \hat{n}=\frac{1}{r}(\hat{p} \times \hat{k}),
$$

(see Fig. 1) where

$$
y=\hat{p} \cdot \hat{k}=\cos \Theta, \quad r=\sqrt{1-y^{2}},
$$

and $\Theta$ represents the top-quark scattering angle. The correlation matrix $C$ can be experimentally accessed through the angular spin correlations of the $t \bar{t}$ leptonic decays - whose directions of flight in the $t$ and $\bar{t}$ rest frames are described, respectively, by the unit vectors $\hat{\ell}_{ \pm}$. These angular spin correlations are determined by properly averaging the products $\xi_{a b}=\cos \theta_{+}^{a} \cos \theta_{-}^{b}$, where we defined the quantities

$$
\cos \theta_{+}^{a}=\hat{\ell}_{+} \cdot \hat{a} \quad \text { and } \quad \cos \theta_{-}^{b}=\hat{\ell}_{-} \cdot \hat{b},
$$

TABLE I. Notation for the labels $a, b$ and corresponding choice of reference axes, following the definitions of Eq. (6).

\begin{tabular}{lcc}
\hline \hline$a, b$ & $\hat{a}$ & $\hat{b}$ \\
\hline$k$ & $\hat{k}$ & $-\hat{k}$ \\
$n$ & $\operatorname{sgn}(y) \hat{n}$ & $-\operatorname{sgn}(y) \hat{n}$ \\
$r$ & $\operatorname{sgn}(y) \hat{r}$ & $-\operatorname{sgn}(y) \hat{r}$ \\
\hline \hline
\end{tabular}

and the labels $a$ and $b \in\{k, n, r\}$ follow the conventions of Table I for the choices of reference axes. Indeed one can show that, in the absence of acceptance cuts, the elements of the $3 \times 3$ matrix $C$ can be expressed as [6]

$$
C_{a b}\left[\sigma\left(m_{t \bar{t}}, \cos \Theta\right)\right]=-9 \frac{1}{\sigma} \int d \xi_{a b} \frac{d \sigma}{d \xi_{a b}} \xi_{a b},
$$

with the residual dependence of the cross section $\sigma$ on $\cos \Theta$ and the invariant mass $m_{t \bar{t}}$ of the top-quark pair system being understood. The integral of Eq. (9) represents precisely the average of the products $\xi_{a b}$ taken over the leptonic angular phase space.

In order to fully take advantage of Eq. (9), the event generation was performed removing any possible kinematic cuts, both in production and decay. The diagonalization of the matrix $C$, needed to test Eq. (4), can be performed as a function of $m_{\bar{t} \bar{t}}$ and $\Theta$.

The result of this procedure is shown in Fig. 2, whose event statistics benefits from the intrinsic initial-state symmetry $\Theta \rightarrow \pi-\Theta$. The binning choice represents the best compromise between the expected event statistics at the LHC and the unavoidable dilution of entanglement effects coming from averaging $\xi$ in bigger portions of phase space.

We can identify in Fig. 2 two regions where (4) holds, one at $m_{t \bar{t}}$ close to threshold, and another at $m_{t \bar{t}} \gtrsim 0.9 \mathrm{TeV}$ and $2 \Theta / \pi \gtrsim 0.7$. Of these two regions, only the one at large $m_{t \bar{t}}$ presents a constructive sum of the $q \bar{q}$ and $g g$ contributions, both giving rise to a top-quark pair state close to a pure, maximally entangled state [6,20], and therefore increase $m_{1}+m_{2}$. In the other region close to threshold, $q \bar{q}$ events produce a mixed state and dilute the $g g$ pure maximally entangled state-even if the $q \bar{q}$ contribution is subdominant in terms of cross section rates. This difference explains the higher values of $m_{1}+m_{2}$ in the top right

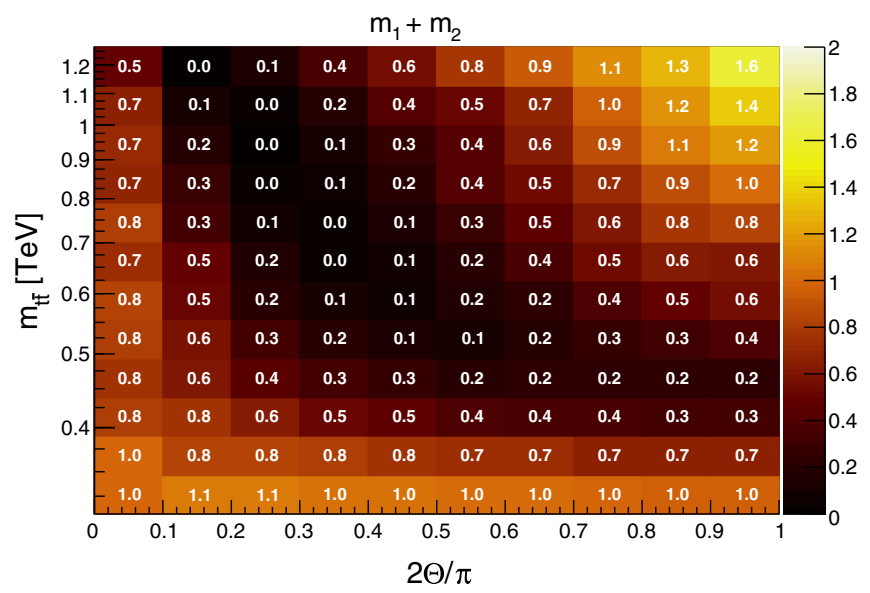

FIG. 2. Values of the observable $m_{1}+m_{2}$ in the phase space of the invariant mass $m_{t \bar{t}}$ vs the scattering angle $\Theta$. The last bins in $m_{t \bar{t}}$ include overflow events. Bins in the upper right corner have the largest values of $m_{1}+m_{2}$ and are selected for testing the violation of the Bell inequality. 
corner of Fig. 2, where $m_{1}+m_{2}$ is expected to reach a value as large as 1.6.

In order to assess to what extent the values of $m_{1}+$ $m_{2}>1$ can realistically be used to prove a violation of the generalized Bell inequalities Eq. (3), we study the impact on their determination of statistical uncertainties due to detector resolution, acceptance, efficiency, and migration effects. To this aim, we reformulate the problem into a statistical test of the null hypothesis $\left\{H_{0}: m_{1}+m_{2} \leq 1\right\}$. We compute the significance of the corresponding outcome at the LHC with $139 \mathrm{fb}^{-1}$, the full Run II luminosity, by performing ten new, independent simulations of the process in Eq. (5), with fast simulation of the ATLAS detector using the DELPHES [24] framework.

We require at least two anti- $k_{t}$ jets with $R=0.4$ and at least one $b$-tagged jet, all with transverse momentum $p_{T}>$ $25 \mathrm{GeV}$ and rapidity $|\eta|<2.5$. Similarly, both $e^{ \pm}$and $\mu^{ \pm}$ leptons are required to have $p_{T}>20 \mathrm{GeV}$ and $|\eta|<2.47$. The neutrino momenta from the dileptonic decay are not directly detectable, since only their sum can be inferred through the missing transverse energy $E_{\text {miss }}^{T}$ of the event. The $t$ and $\bar{t}$ momenta need thus to be reconstructed using the neutrino weighting technique [25]. With this method, the sums of the momenta of the candidate reconstructed neutrinos, charged leptons, and candidate $b$ jets are constrained to satisfy four equations on the invariant mass of the two candidate $W$ bosons and top quarks. The possible solutions of this unconstrained system are assigned a weight $w$. The solution which maximizes $w$ is eventually used to reconstruct the $t$ and $\bar{t}$ momenta for that event. This procedure allows us to determine the reconstructed distributions which are eventually corrected for detector resolution and acceptance effects using a simplified unfolding procedure. The good agreement between our response matrix and those published for comparable processes [26] shows that migration effects have been properly simulated.

Tuning such pseudoexperiments to have a statistics equal to the one expected with present LHC luminosity, we can take the resulting standard deviations $s_{i}$ on $m_{1}+m_{2}$ as the predicted statistical uncertainty, with detector effects included. In testing the hypothesis, we use a standard $\chi^{2}$ statistical test,

$$
\chi^{2}=\sum_{i} \frac{\left(1-m_{1}^{i}-m_{2}^{i}\right)^{2}}{s_{i}^{2}},
$$

where the sum runs over the set of bins that maximize the standard model expected significance for $m_{1}+m_{2}>1$.

We find that, under such conditions, the null hypothesis and the violation of Eq. (3) can be assessed at the 98\% C.L. with present Run II luminosity. Moreover, after rescaling this result by the projected luminosity of the LHC full Run III, we expect that it will be possible to test the violation at the $99.99 \%$ C.L. ( $4 \sigma$ significance).

While systematic uncertainties associated with the unfolding procedure itself are known to be negligible
[26], the results hereby presented do not include other theoretical and experimental systematic effects, the inclusion of which is beyond the scope of the present Letter since it would require a more detailed simulation both of the detector and of the collisions conditions, which is only possible for the experimental collaborations.

Conclusions.-We have shown that the measurement of a single, suitable defined observable of the top-quark pair system can be used to ascertain quantum correlations among the spins of two quarks and in turn test a Bell inequality with the data already collected at the LHC. This test can provide clear evidence for quantum mechanics in an energy range never explored before.

Testing Bell inequalities at high-energy colliders differs from the more familiar tests performed using quantum optics experiments. In the latter, the request of Bell locality can be easily achieved by using spin polarization measurements that are spacelike separated; these measurements cannot influence each other, so that the two events have independent statistics. It is not possible to follow the same procedure in the case of the top-quark system because of the obvious restrictions of the detectors employed at the LHC.

Nevertheless, there are advantages in studying Bell's inequalities in high-energy settings along the lines discussed in this Letter. In quantum optics tests, to avoid socalled loopholes connected to the lack of control of the number of pairs produced that actually impinge in the detectors, and other inevitable inefficiencies, one is generally forced to use inequalities more involved than (2). Although loophole-free tests of Bell inequalities have been recently performed in quantum optics $[27,28]$, none of these problems affect the (indirect) Bell test presented above, as it reduces to the study of the spin correlation matrix $C$, without the need of any a priori commitment about efficiencies of detectors.

We believe that our results will stimulate additional analyses to test Bell inequalities by means of the actual experimental data collected at LHC and motivate further investigations on other possible tests of quantum mechanics at high-energy colliders.

We thank M. Pinamonti for help on the data analysis. M. F. is affiliated to the Physics Department of the University of Trieste, the Scuola Internazionale Superiore di Studi Avanzati, and the Institute for Fundamental Physics of the Universe, Trieste, Italy-the support of which is gratefully acknowledged. R.F. acknowledges that his research was conducted within the framework of the Trieste Institute for Theoretical Quantum Technologies.

[1] R. Horodecki, P. Horodecki, M. Horodecki, and K. Horodecki, Rev. Mod. Phys. 81, 865 (2009).

[2] J. S. Bell, Phys. Phys. Fiz. 1, 195 (1964).

[3] J.S. Bell, Speakable and Unspeakable in Quantum Mechanics (Cambridge University Press, Cambridge, England, 
1987-2004); M. Redhead, Incompleteness, Nonlocality and Realism (Clarendon Press, Oxford, 1987); Quantum [Un] Speakables II: Half a Century of Bell's Theorem, edited by R. A. Bertlmann and A. Zeilinger (Springer, Berlin, 2017); N. Brunner et al., Rev. Mod. Phys. 86, 419 (2014).

[4] J. F. Clauser and A. Shimony, Rep. Prog. Phys. 41, 1881 (1978).

[5] W. Bernreuther, A. Brandenburg, Z. G. Si, and P. Uwer, Phys. Rev. Lett. 87, 242002 (2001); W. Bernreuther and Z. G. Si, Nucl. Phys. B837, 90 (2010); Phys. Lett. B 725, 115 (2013); 744, 413(E) (2015); G. Mahlon and S. J. Parke, Phys. Rev. D 81, 074024 (2010).

[6] W. Bernreuther, D. Heisler, and Z. G. Si, J. High Energy Phys. 12 (2015) 026.

[7] G. Aad et al. (ATLAS Collaboration), Phys. Rev. Lett. 108, 212001 (2012); 111, 232002 (2013); S. Chatrchyan et al. (CMS Collaboration), Phys. Rev. Lett. 112, 182001 (2014); G. Aad et al. (ATLAS Collaboration), Phys. Rev. D 90, 112016 (2014).

[8] G. Aad et al. (ATLAS Collaboration), Phys. Rev. Lett. 114, 142001 (2015); M. Aaboud et al. (ATLAS Collaboration), J. High Energy Phys. 03 (2017) 113.

[9] A. M. Sirunyan et al. (CMS Collaboration), Phys. Rev. D 100, 072002 (2019).

[10] J. F. Clauser, M. A. Horne, A. Shimony, and R. A. Holt, Phys. Rev. Lett. 23, 880 (1969).

[11] J. F. Clauser and M. A. Horne, Phys. Rev. D 10, 526 (1974).

[12] R. Horodecki, P. Horodecki, and M. Horodecki, Phys. Lett. A 200, 340 (1995).

[13] M. Genovese, Phys. Rep. 413, 319 (2005).

[14] N. A. Tornqvist, Found. Phys. 11, 171 (1981).
[15] F. Benatti and R. Floreanini, Phys. Rev. D 57, R1332 (1998); Mod. Phys. Lett. A 14, 1519 (1999); Eur. Phys. J. C 13, 267 (2000); R. A. Bertlmann, W. Grimus, and B. C. Hiesmayr, Phys. Lett. A 289, 21 (2001).

[16] S. Banerjee, A. K. Alok, and R. MacKenzie, Eur. Phys. J. Plus 131, 129 (2016).

[17] A. Acin, J. I. Latorre, and P. Pascual, Phys. Rev. A 63, 042107 (2001).

[18] S. P. Baranov, J. Phys. G 35, 075002 (2008); S. Chen, Y. Nakaguchi, and S. Komamiya, Prog. Theor. Exp. Phys. (2013), 063A01; C. Qian, J. L. Li, A. S. Khan, and C. F. Qiao, Phys. Rev. D 101, 116004 (2020).

[19] S. Banerjee, A. K. Alok, R. Srikanth, and B. C. Hiesmayr, Eur. Phys. J. C 75, 487 (2015).

[20] Y. Afik and J. R. M. de Nova, Eur. Phys. J. Plus 136, 907 (2021).

[21] A. Cervera-Lierta, J. I. Latorre, J. Rojo, and L. Rottoli, SciPost Phys. 3, 036 (2017).

[22] J. Alwall et al., J. High Energy Phys. 07 (2014) 079.

[23] R. D. Ball et al., Nucl. Phys. B867, 244 (2013).

[24] J. de Favereau et al. (DELPHES 3 Collaboration), J. High Energy Phys. 02 (2014) 057.

[25] B. Abbott et al. (D0 Collaboration), Phys. Rev. Lett. 80, 2063 (1998).

[26] M. Aaboud et al. (ATLAS Collaboration), Eur. Phys. J. C 80, 754 (2020); S. Chatrchyan et al. (CMS Collaboration), Phys. Rev. Lett. 112, 182001 (2014).

[27] B. Hensen, H. Bernien, A. E. Dreau, A. Reiserer, N. Kalb, M. S. Blok, J. Ruitenberg, R. F. L. Vermeulen, R. N. Schouten and C. Abellan et al., Nature (London) 526, 682 (2015).

[28] M. Giustina et al., Phys. Rev. Lett. 115, 250401 (2015). 